\title{
Emerging solar systems in view
}

\section{The Infra-Red Astronomy Satellite has produced evidence of the early stages of planet formation around a star. It is not the only evidence of solar systems other than our own.}

WITH a discovery as provoking to the imagination as a possible new solar system (will it be like ours? will it have life?) it is almost inevitable that a crop of excited and over-simplified stories will appear in the media ("Hullo out there" began one editorial in a Canadian newspaper). Just as inevitably, there will be some who will ask: why should we be excited, surely it is no surprise? So how should one react to observations from Hawaii that indicate, some say, a giant planet orbiting T-Tauri; to recent measurements with the new Japanese millimetre-wave radiotelescope at Nobeyama, near Tokyo, which seem to show swirling gas clouds around a star in Orion; and to this month's results from the Infra-Red Astronomy Satellite, IRAS, suggesting something similar (but much smaller) around the star Vega?

Certainly it is not surprising that stars probably form from collapsing gas clouds, and that in these clouds subsidiary vortices might form planets. But the theory of the process is uncertain, and it is not known how frequently planets form. Observations are needed but have been difficult for various reasons - paramount among them being that planets are small and dark; that planetary orbits are expected to be too small to resolve readily from Earth even if the planet is bright; and that a forming system is inevitably shrouded in dust. Hence there would be great delight if more planets, or solar systems in the process of formation, could be found and studied. (And such observations - if there were enough of them - would also eventually bear on the vexed calculations of the probability of life in the Universe, by increasing the accuracy with which the formation of an Earth-like planet can be predicted.)

Therefore, there is great interest in discovering solar systems at any stage of their development. In practice this probably means the formative stage, when the cloud and protoplanets should be hot and radiating. Such conditions are the basis of the recent measurements, although one should not forget the older technique, independent of temperatures, based on observing a wobble in the orbit of a star due to its planets.

Unfortunately that technique is only applicable in favourable circumstances with close stars. Such was the case with Barnard's star, the closest in the northern sky, whose tiny wobble of a few hundredths of an arc second has long been thought to indicate the presence of (probably two) planets.

The latest measurements, however, are thought to represent direct observations of clouds, condensing or not. The most recent is the IRAS observation of Vega, which came about by chance. While Vega was being used for calibration it was observed to be 10-20 times brighter than it should be at wavelengths of $60-1,000 \mu \mathrm{m}$, assuming the star radiates like a black body at its optically-defined temperature of $9,700 \mathrm{~K}$. The star is also extended at these long wavelengths, to about 20 arc seconds - or around 80 astronomical units ( $1 \mathrm{AU}$ is the radius of the Earth's orbit about the Sun) at the 26-light-year distance of Vega. The IRAS team's explanation of these observations invokes a ring of dust with grains probably greater than $1 \mathrm{~mm}$ diameter (compared with interstellar grains of $0.1 \mu \mathrm{m}$ ) orbiting Vega. The dust would be heated to 90 $\mathrm{K}$ by Vega's radiation and would re-radiate predominantly at the wavelengths detected by IRAS.

Vega is a hot bright star which has a predicted lifetime of around 300 million years (compared with the Sun's 10,000 million), so the cloud can be interpreted as the "early stages" of a solar system although Vega might be dead before the system can be completed.

The possibility of a "planet" near the star T-Tauri, one of the best examples of a very young star, or protostar, was raised shortly before the IRAS observations by R.B. Hanson, B.F. Jones and D.N.C. Lin (Astrophys. J. Lett. 270, L27; 1983). They base their suggestion on observations by H.M. Dyck, T. Simon and B. Zuckerman (Astrophys. J. Lett. 255, L103; 1982) of a low-luminosity infrared source near T-Tauri. When measured by Dyck et al. with a University of Hawaii telescope, using speckle interferometry to separate close objects, the infrared luminosity was lower by a factor of ten than the lowest luminosity infrared point source previously recorded.

Hanson et al. suggest that the infrared luminosity is the heat from a very large condensing protoplanet. But Zuckerman professes himself unconvinced. The controversy will probably be unresolved until there is better theoretical understanding of how much and in what wavelengths a protoplanet should radiate.

Between the Hawaiian and IRAS observations come the Japanese millimetre wave observations (still unpublished) that were interpreted as evidence of protoplanetary disks - the condensing gas clouds in their final stages. Using fine molecular lines emitted by carbon monosulphide, a group headed by Dr Norio Kaifu claimed observations of Doppler shifts caused by the rotation of a disk around a star in Orion. But the resolution of the telescope (about 20 arc seconds) is at the moment poor by optical standards (it should improve 20 -fold when an interferometer system is complete at the end of next year). So the discovered Orion disk is large $(40,000 \mathrm{AU}$ : the radius of our Solar System is about 20 $\mathrm{AU})$ and the central star very bright and large (about 300 solar masses). Therefore the chances are this cloud will be blown away before planets can form.

The group also found other candidate disks: one around the star L1551 in Taurus was only $15,000 \mathrm{AU}$ across, Kaifu says. In both cases, measurements with other techniques have shown matter streaming away from the central stars along a line perpendicular to the disk plane: this agrees with predictions that the bright protostar will easily be able to "blow away" matter perpendicular to the disk but not in its plane, where the matter is too dense.

Thus the Japanese may indeed have found the first evidence for large planetary disks, and it would be interesting if IRAS could turn to check the measurements. Unfortunately the orbital observatory does not have the spectral resolution necessary to detect disk rotation (by Doppler shift). With its high angular resolution, however, IRAS may be able to add structural and temperature measurements of the putative disks - and, conceivably, detect hotspots that might indicate the early formation of planets.

At the same time IRAS can safely be predicted to discover more candidate solar systems during its general sky survey - to be repeated three times before the observatory runs out of cooling helium at the end of the year. And the Japanese are planning to add an interferometer to their telescope to improve its resolution 20 -fold.

Not to be outdone, the angular resolution of the speckle interferometer on the University of Hawaii telescope is to be sharpened. Further down the line are proposed ground-based telescopes with resolutions close to one hundredth of an arc second, offering hope of seeing planets or protoplanetary disks.

There should be much more evidence of solar systems to come in the next months and years.

Robert Walgate 